\title{
EQUAÇÕES ALOMÉTRICAS PARA ESTIMATIVA DE BIOMASSA E CARBONO EM ÁRVORES DE REFLORESTAMENTOS DE RESTAURAÇÃO ${ }^{1}$
}

\author{
Dirceu Lucio Carneiro de Miranda², Antônio Carlos Galvão de Melo³ e Carlos Roberto Sanquetta ${ }^{4}$ \\ RESUMO - Este estudo teve como objetivos desenvolver e ajustar modelos alométricos para estimativa da \\ biomassa seca e do carbono total em árvores plantadas em áreas de restauração florestal. Os dados dos ajustes \\ são provenientes de 107 árvores de 44 espécies plantadas na região do Médio Vale do Paranapanema, SP, localizado \\ nos Biomas Mata Atlântica e Cerrado. A biomassa seca e a massa de carbono foram obtidas por meio de amostragem \\ destrutiva da parte aérea e subterrânea das árvores. Para o teste e ajuste dos modelos foi realizada a estratificação \\ do conjunto inicial dos dados em ritmos de crescimento das espécies amostradas. Os ajustes foram feitos usando-se \\ oito modelos lineares de cada variável dependente e dois obtidos pelo processo Stepwise-Forward. Os melhores \\ modelos para estimativa da biomassa seca e carbono orgânico apresentaram coeficientes de determinação ajustado \\ acima de 0,95 e erros-padrão percentuais abaixo de $32 \%$. Os modelos baseados nos ritmos de crescimento das \\ espécies mostraram melhores resultados estatísticos, atingindo $\mathrm{R}^{2}=0,985$ e Syx\% $=16,15$ para biomassa seca \\ das espécies de crescimento lento. Modelos provenientes do procedimento Stepwise geraram as melhores equações \\ para as estimativas de biomassa seca e carbono total, e a estratificação dos dados dos diferentes ritmos de crescimento \\ das espécies amostradas mostrou-se que foi adequada para melhorar o desempenho dos modelos.
}

Palavras-chave: Cerrado, Mata Atlântica e Sequestro de carbono.

\section{ALLOMETRIC EQUATIONS FOR BIOMASS AND CARBON STOCK ESTIMATION IN TREES OF FOREST RESTORATION AREAS}

\begin{abstract}
The objective of this study was to develop and to fit allometric models to estimate dry biomass and total carbon in trees planted in forest restoration areas. Fit data are from 107 trees of 44 species planted in Médio Paranapanema Vale, SP, Brazil, located in the Atlantic Forest and Cerrado Biomes. Dry biomass and carbon mass were obtained by destructive sampling taken from aerial and underground sections of the trees. For model test and fitting, stratification of the initial data set was made in growth rhythms of the sampled species. Adjust was done by using eight linear models of each dependent variable and two were obtained from Stepwise-Forward method. The best models to estimate dry biomass and carbon stock presented adjusted determination coefficient above 0.95 and standard error below $32 \%$. Models based on growth rate of the species presented the best statistical results, reaching $R^{2}=0.985$ and Syx $\%=16.15$ for dry biomass of low growth species. Models created by Stepwise procedure produced the best equations for estimates of dry biomass and total carbon, and data stratification of different growth rates of the sampled species was suitable for improving performance of the models.
\end{abstract}

Keywords:Savana, Atlantic Rain Forest and Carbon sequestration.

\section{INTRODUÇÃO}

Associa-se às florestas de restauração uma série de serviços ambientais, como a proteção e recuperação da biodiversidade através da melhoria das características da paisagem e do restabelecimento de processos ecológicos e a proteção do solo e da água (ENGEL; PARROTA, 2003). Atualmente, em função das ameaças advindas do aquecimento global, a fixação do carbono atmosférico passou também a ser apontado como importante benefício proporcionado por essas florestas (MELO; DURIGAN, 2006).

\footnotetext{
${ }^{1}$ Recebido em 14.05.2009 e aceito para publicação em 18.04.2011.

${ }^{2}$ Universidade Federal de Mato Grosso, UFMT, Brasil. E-mail: >mirandaufpr@yahoo.com.br>.

${ }^{3}$ Instituto Florestal do Estado de São Paulo, IF, Brasil. E-mail: <acgmelo@gmail.com>.

${ }^{4}$ Departamento de Ciências Florestais, Universidade Federal do Paraná, UFPR, Brasil. E-mail: <sanquetta@ufpr.br>.
} 
O interesse pelos estudos acerca da biomassa e do conteúdo de carbono estocado nos diferentes biomas brasileiros é crescente e podem ser citados estudos para o Cerrado (DELITTI et al., 2001; REZENDE et al., 2006; SALLIS et al., 2006;), para a Mata Atlântica (CALDEIRA et al., 2004; SANTOS et al., 2006) e para a Amazônia (NELSON et al., 1999; CHAMBERS et al., 2001; HIGUCHI et al., 2004).

Estudos que quantifiquem a capacidade de fixação de carbono pelas florestas de restauração ainda são escassos, podendo ser citados Melo e Durigan (2006), que apresentaram estimativa de estoque e incremento de carbono em reflorestamentos heterogêneos com idades variando de um a 28 anos.

Uma das maneiras de se conhecer o estoque de carbono em árvores é por meio do desenvolvimento de modelos matemáticos para sua estimativa (SANQUETTA et al., 2004), porém, em razão das dificuldades logísticas, as experiências com modelagem envolvendo espécies florestais nativas têm-se restringido aos modelos de volume. No caso dos plantios de restauração, inexistem estudos envolvendo modelagem da biomassa e do carbono, deixando assim uma lacuna de informações a respeito do potencial dessas florestas como sumidouros de carbono.

Este estudo teve como objetivos gerar e ajustar equações para estimativa de biomassa e carbono em árvores de reflorestamentos de restauração.

\section{MATERIAL E MÉTODOS}

As árvores amostradas foram selecionadas em nove diferentes reflorestamentos de restauração florestal ou de experimentos florestais que contavam com diversos arranjos espaciais (Tabela 1), porém todos realizados com espécies nativas regionais e localizados no Médio Vale do Paranapanema, SP.

O clima da região, de acordo com o sistema da classificação de Köppen, é do tipo Cfa, ou seja, mesotérmico com temperaturas inferiores a $18^{\circ} \mathrm{C}$ nos meses mais frios e de $22{ }^{\circ} \mathrm{C}$ nos meses mais quentes, sujeito a geadas esporádicas. A precipitação média anual é de 1.350 mm (SILVEIRA; DURIGAN, 2004).

Na região ocorriam, originalmente, a Floresta Estacional Semidecidual e o Cerrado e predominavam os solos profundos, argilosos ou de textura média, relevo suave-ondulado, com uso predominante para culturas anuais e cana-de-açúcar (MELO; DURIGAN, 2006).
Tabela 1 - Caracterização das áreas de amostragem de árvores de reflorestamentos com espécies nativas utilizadas para desenvolvimento de modelos alométricos para estimativa de biomassa e carbono.

Table 1 - Characterization of the areas of sampling of trees in reforestation withnative species used for the development of allometric models to estimate biomass and carbon.

\begin{tabular}{lccc}
\hline $\begin{array}{l}\text { Coordenadas } \\
\text { UTM }\end{array}$ & $\begin{array}{c}\text { Idade } \\
\text { (anos) }\end{array}$ & $\begin{array}{c}\text { Densidade } \\
\text { (árvores.ha }^{-1} \text { ) }\end{array}$ & $\begin{array}{c}\text { Posicionamento } \\
\text { na paisagem }\end{array}$ \\
\hline $560800 / 7504043$ & 36 & 2500 & interflúvio \\
$5600735 / 7503123$ & 35 & 1250 & interflúvio \\
$560642 / 7503224$ & 35 & 1111 & interflúvio \\
$558432 / 7501759$ & 31 & 1667 & interflúvio \\
$558156 / 7501025$ & 19 & 1667 & interflúvio \\
$542595 / 7479011$ & 16 & 2222 & ripário \\
$545195 / 7487033$ & 8 & 1667 & ripário \\
$527798 / 7476935$ & 7 & 1667 & ripário \\
$561562 / 7499536$ & 5 & 1667 & ripário \\
\hline
\end{tabular}

Os dados básicos para o desenvolvimento deste estudo foram provenientes de 107 árvores de 44 espécies (Tabela 2). Os diâmetros à altura do peito variaram de 4,5 a $57,5 \mathrm{~cm}$ e as alturas, de 3,9 a $22,6 \mathrm{~m}$.

Sabe-se que a densidade da madeira é variável importante na modelagem da biomassa em árvores (BROWN et al., 1989), porém não se encontram, na literatura, valores de densidade para todas as espécies amostradas neste estudo. Dessa forma, optou-se por classificá-las de acordo com o ritmo de crescimento (Tabela 2) em rápidas, médias e lentas, conforme a classificação elaborada por Durigan et al. (2004).

Cada árvore teve sua biomassa separada em quatro compartimentos:

- Raízes: todo o material radicular com diâmetro maior que $1 \mathrm{~cm}$.

- Fuste: material localizado entre o colo e o ponto de inversão morfológica.

- Galhos: material lenhoso acima do ponto de inversão morfológica até o diâmetro mínimo de $1 \mathrm{~cm}$.

- Folhagem: folhas e ramos finos (abaixo de $1 \mathrm{~cm}$ de diâmetro).

As árvores, logo que abatidas, tiveram seus compartimentos separados e pesados no campo em dinamômetro com capacidade para 200 kg e precisão de 50 g.

Para determinação do teor de massa seca, foi retirada uma amostra de cada compartimento, exceto do fuste, de onde foram colhidas três amostras: uma de base

Revista Árvore, Viçosa-MG, v.35, n.3, Edição Especial, p.679-689, 2011 
(junto ao colo da árvore), uma a 50\% da altura e a última no topo do fuste. Essas amostras, logo depois de coletadas, foram pesadas em uma balança semianalítica com capacidade para $5 \mathrm{~kg}$ e precisão de 0,1 g e posteriormente secas em estufa a $60{ }^{\circ} \mathrm{C}$ para folhas e $85^{\circ} \mathrm{C}$ para as demais amostras até a estabilização do peso, quando eram novamente pesadas.
A biomassa total dos diferentes compartimentos das árvores foi calculada em porcentagem de massa de matéria seca, obtida a partir das amostras, por meio da fórmula:

Teor de matéria seca $=$ (Peso de matéria seca/ Peso de matéria verde) * 100

Tabela 2 - Espécies nativas amostradas em reflorestamentos ciliares para desenvolvimento de modelos alométricos de estimativa de biomassa e carbono.

Table 2 - Native species sampled from riparian reforestation for development of allometric models to estimate biomass and carbon.

\begin{tabular}{|c|c|c|}
\hline Espécie & Ritmo de crescimento & $\mathrm{n}$ \\
\hline Acacia polyphyla D.C. & Médio & 1 \\
\hline Anadenanthera falcata (Benth.) Speg. & Rápido & 8 \\
\hline Anadenanthera macrocarpa (Benth.) Brenan & Rápido & 5 \\
\hline Astronium graveolens Jacq. & Lento & 1 \\
\hline Baulforodendron riedelianum (Engl.) Engl. & Lento & 3 \\
\hline Calophylum brasiliense Cambess. & Médio & 2 \\
\hline Casearea sylvestris Sw. & Médio & 1 \\
\hline Cecropia pachystachia Trécul & Rápido & 1 \\
\hline Cedrela fissilis Vell. & Médio & 1 \\
\hline Ceiba speciosa A. St.-Hill. & Médio & 3 \\
\hline Centrolobium tomentosum Guill. ex Benth. & Médio & 1 \\
\hline Colubrina glandulosa Perkins & Médio & 3 \\
\hline Copaifera langsdorfii Desf & Médio & 1 \\
\hline Cordia ecalyculata Vell. & Rápido & 1 \\
\hline Croton floribundus (L.) Spreng. & Rápido & 2 \\
\hline Croton urucurana Baill. & Rápido & 3 \\
\hline Cupania vernalis Camb. & Médio & 1 \\
\hline Cyclolobium vecchi A. Samp. Ex Hoehne. & Lento & 4 \\
\hline Cytharexyllum myrianthum Cham. & Rápido & 5 \\
\hline Dipteryx alata Vogel & Médio & 3 \\
\hline Enteroplobium contortisiliquum (Vell.) Morong & Rápido & 2 \\
\hline Ficus guaranitica Chodat & Médio & 1 \\
\hline Genipa americana L. & Médio & 1 \\
\hline Gochnatia polymorpha (Less.) Cabreira & Médio & 2 \\
\hline Guazuma ulmifolia Lam. & Rápido & 1 \\
\hline Helietta apiculata Benth. & Lento & 1 \\
\hline Heliocarpus popayanensis Kunth. & Rápido & 3 \\
\hline Hymenaea courbaril var. stilbocarpa (Hayne) Lee et Lang. & Lento & 1 \\
\hline Inga uruguensis HooK. e Arn. & Rápido & 3 \\
\hline Myracrodruon urundeuva Allemao & Lento & 5 \\
\hline Myroxylon peruiferum L. f. & Lento & 2 \\
\hline Nectandra megapotamica (Spreng.) Mez & Médio & 2 \\
\hline Peltophorum dubium (Spreng.) Taub. & Rápido & 5 \\
\hline Plathymenia reticulata Benth. & Médio & 5 \\
\hline Platypodium elegans Vogel & Lento & 4 \\
\hline Psidium guajava $\mathrm{L}$. & Lento & 1 \\
\hline Schizolobium parahyba (Vell.) S. F. Blake & Rápido & 2 \\
\hline Tabebuia heptaphylla (Vell.) Toledo & Médio & 4 \\
\hline Tabebuia ochraceae (Cham.) Standl. & Lento & 1 \\
\hline Tapirira guianensis Aubl. & Rápido & 3 \\
\hline Terminalia glabrescens Mart. & Médio & 2 \\
\hline Triplaris brasiliana Cham. & Médio & 1 \\
\hline Vitex montevidensis Cham. & Médio & 2 \\
\hline Zanthoxylum rhoifolium Lam. & Médio & 3 \\
\hline
\end{tabular}


Para cada uma das amostras retiradas foi realizada a determinação dos teores de carbono por meio do equipamento analisador de carbono em sólidos da marca Leco C-144. O peso de carbono total de cada árvore foi conseguido multiplicando-se o peso da biomassa de matéria seca de cada compartimento pelo respectivo teor de carbono.

A biomassa de matéria seca e o peso de carbono total de cada árvore foram obtidos pelo somatório dos pesos de cada compartimento.

Para cada indivíduo foram medidos o diâmetro à altura do peito (DAP) e altura total, enquanto a idade foi determinada através de informação prestada diretamente pelos proprietários das áreas em restauração. Para as árvores que eram bifurcadas, fato comum em reflorestamentos de restauração, foram tomadas as medidas de DAP de todos os fustes.

Para o desenvolvimento e ajuste dos modelos, foram consideradas as seguintes variáveis:

- Diâmetro quadrático $(D q)=\sqrt{d 1^{2}+d 2^{2}+d 3^{2}+\ldots d n^{2}}$, em que $d=$ diâmetro dos fustes de cada árvore (MAcDICKEN et al., 1991);

- Área transversal $(G)$ = Soma das áreas transversais dos fustes de uma mesma árvore medido a 1,30 m do solo, expressa em $\mathrm{m}$;

- Maior diâmetro $(D m)=$ maior diâmetro a 1,3 m encontrado em uma mesma árvore, expresso em cm;

- Altura total da árvore expressa em m (h); e

- Idade do plantio em anos (Id).

O ajuste de equações para biomassa de matéria seca e carbono foi realizado em oito modelos lineares selecionados na literatura, listados a seguir:

(1) $\ln Y=b_{0}=b_{1} \ln D q+b_{2}, \ln h$ (Modelo de Schumacher - Hall)

(2) $Y=b_{0}+b_{1}\left(D q^{2} h\right)$ (Modelo de Spurr)

(3) $Y=b_{0}+b_{1} D q+b_{2} D q^{2}+b_{3} h+b_{4} D q^{2} h$ (Modelo de Meyer)

(4) $b_{0}+b_{1} D q^{2}+b_{2} h^{2}+b_{3} D q^{2} h$ (Modelo de Stoate)

(5) $Y=b_{0}+b_{1} D q+b_{2} D q^{2} h$ (HIGUCHI; CARVALHO JR., 1994)

(6) $Y=b_{0}+b_{1} D q+b_{2} D q^{2}+b_{3} D q^{2} h$ (HIGUCHI; CARVALHO JR., 1994)
(7) $Y=b_{0}+b_{1} D q^{2}+b_{2} D q^{2} h$ (SANQUETA et al., 2001)

(8) $Y=b_{0}+b_{1} D q^{2}+b_{2} D q^{2} h+b_{3} D q h_{2}+b_{4} h^{2}$ (Modelo de Naslund)

em que:

$\mathrm{Y}=$ BST (Biomassa de matéria Seca Total) ou CT(Carbono Total); e

Ln = logaritmo neperiano.

A geração de modelos por seleção das variáveis significativas foi realizada por meio do processo Stepwise (DRAPPER; SMITH, 1980). Nesse processo foi montada uma matriz de correlação simples usando-se 88 variáveis independentes, sendo estas puras, transformadas ou combinadas, visando atingir melhor correlação entre as variáveis dependentes e independentes. A partir desse método foram gerados mais dois modelos para cada variável analisada (biomassa de matéria seca e carbono), um com a variável dependente transformada para logaritmo neperiano, sendo neste estudo denominados modelos 9 e 10 .

Com a utilização desses procedimentos foram geradas e ajustadas equações para o conjunto total de árvores e para os três diferentes ritmos de crescimento.

Os critérios para a seleção dos melhores modelos foram os coeficientes de determinação ajustado $\left(\mathrm{R}^{2}{ }_{\mathrm{aj}}\right)$, os erros-padrão de estimativa em percentagem (Syx\%) e análise gráfica dos resíduos.

Para os modelos em que a variável dependente sofreu transformação logarítmica nas suas unidades originais, o coeficiente de determinação foi recalculado, a fim de permitir a comparação com os coeficientes obtidos nos demais modelos. Para tanto, foi realizada a correção da discrepância logarítmica, multiplicando-se o peso estimado de cada árvore pelo Fator de Correção de Meyer, calculado pela fórmula:

$$
\mathrm{FC}=\mathrm{e}^{0,5 \mathrm{Syx}}
$$

em que:

$\mathrm{e}=2,718281828$; $\mathrm{e}$

Syx = erro-padrão da estimativa.

Ao estratificar os dados pelos diferentes ritmos de crescimento, os valores absolutos também sofreram mudanças nos ajustes. No entanto, nos modelos 1 e 10, em que variável dependente sofreu transformação,

Revista Árvore, Viçosa-MG, v.35, n.3, Edição Especial, p.679-689, 2011 
foi necessário o recálculo do erro-padrão residual, utilizando, assim, os pesos corrigidos anteriormente pelo Fator de Correção de Meyer.

\section{RESULTADOS E DISCUSSÃO}

Os modelos ajustados (Tabela 3) ou gerados (Tabela 4) para as árvores de reflorestamentos de restauração mostraram, de forma geral, bons índices de ajuste e precisão, podendo ser comparados com outros trabalhos sobre a estimativa da biomassa e carbono para conjuntos de diversas espécies.

Outros autores têm utilizado modelos matemáticos para estimativa da biomassa e do carbono em florestas que envolvem ampla variedade de espécies. Rezende et al. (2006) encontraram coeficientes de determinação acima de 0,93 e erro-padrão da estimativa entre 25,03 e 28,09\%, em estudo em que foram amostradas 174 árvores de 49 espécies de cerrado.

Higuchi et al. (1998), testando 14 modelos para estimar a biomassa verde na Floresta Amazônica, obtiveram coeficientes de determinação variando entre 0,874 e 0,99. Ainda na Floresta Amazônia, Santos et al. (2001) testaram 17 modelos para estimativa da biomassa total, em que o melhor deles apresentou $\mathrm{R}^{2}=0,9751$. Todos os estudos citados tiveram resultados, em termos de qualidade de ajustes expressos pelos coeficientes de determinação, muito próximos dos obtidos neste estudo.

Silva e Sampaio (2008), ao ajustarem modelos para estimativa de biomassa aérea de árvores de nove espécies da caatinga, obtiveram $\mathrm{R}^{2}=0,946$ para espécies de maior porte e $\mathrm{R}^{2}=0,819$ para árvores de menor porte.

As equações provenientes de processo Stepwise (Tabela 4) resultaram nas melhores estimativas para as variáveis de interesse, com exceção do ajuste para estimativa da biomassa de matéria seca das espécies de crescimento lento, em que a melhor equação foi ajustada pelo modelo 2 (Tabela 3).

Quando se considera o conjunto de todas as árvores amostradas, os melhores modelos ajustados utilizando variáveis combinadas de diâmetro, idade e altura foram os modelos $10\left(\mathrm{R}^{2}=0,954\right.$ e Syx\% = 31,72) para estimativa de biomassa e $9\left(\mathrm{R}^{2}=0,957\right.$ Syx\% $\left.=31,19\right)$ para carbono.

A estratificação do conjunto de dados por ritmo de crescimento mostrou-se adequada, pois os ajustes obtidos com essa estratégia mostraram melhor desempenho, com diminuição do erro- padrão da estimativa e aumento do coeficiente de determinação.
Baseado nos ritmos de crescimento das espécies, os melhores modelos para estimar a biomassa de matéria seca e o carbono total foram, respectivamente, os modelos $9\left(R^{2}=0,979 ;\right.$ Syx $\left.\%=28,0\right)$ e $9\left(R^{2}=0,971 ;\right.$ Syx $\left.\%=28,54\right)$ para as espécies de crescimento rápido, $9\left(\mathrm{R}^{2}=0,973\right.$; Syx\% $=19,64)$ e $9\left(R^{2}=0,973 ;\right.$ Syx\% $\left.=20,04\right)$ para as espécies de crescimento médio, $2\left(\mathrm{R}^{2}=0,985\right.$; Syx\% = 16,15) e $10\left(R^{2}=0,979 ;\right.$ Syx $\left.\%=18,8\right)$ para as espécies de crescimento lento.

O ajuste para estimativa de biomassa de matéria seca, por exemplo, foi consideravelmente melhorado pela estratificação, quando se compara o erro-padrão da estimativa obtido no modelo que considerou todas as árvores ( $\mathrm{Syx} \%=31,72 \%)$ com o modelo obtido exclusivamente para as espécies de crescimento lento $($ Syx\% $=16,15 \%)$.

A estratificação em duas classes de diâmetro foi utilizada por Higuchi et al. (2004) em estudo de modelagem da biomassa em árvores de formações primárias daAmazônia Central, como forma de aumentar a qualidade dos indicadores estatísticos de ajuste e precisão dos modelos e, como neste trabalho, os resultados foram satisfatórios.

Além do cálculo do $\mathrm{R}^{2}$ e Syx\%, levou-se em consideração a análise gráfica dos resíduos de todos os modelos, sendo apresentados os gráficos dos melhores modelos. Aqueles modelos que apresentaram tendências em suas estimativas, mesmo tendo parâmetros de $\mathrm{R}^{2}$ e Syx\% superiores, não foram apresentados como os melhores. Dessa forma, a análise gráfica dos resíduos (Figuras 1 e 2) foi determinante para a escolha dos melhores ajustes no caso da estimativa de biomassa de matéria seca do conjunto total de dados e das árvores de crescimento lento. Neste último caso, a análise gráfica mostrou que os resultados obtidos por meio do processo Stepwise apresentaram tendências a superestimativas.

Mesmo para o conjunto total de dados, de todas as 44 espécies, os resultados não se apresentaram inferiores àqueles obtidos em nível de uma única espécie, como no estudo de Scolforo et al. (2004), que testaram 12 modelos para estimativa da biomassa de matéria seca total de Eremanthus erythropappus (DC.) MacLeish e obtiveram, como melhor resultado, $\mathrm{R}^{2}=0,971$. Em árvores de Mimosa scabrella Benth. Urbano (2007), obtiveram-se $\mathrm{R}^{2}=0,960$ e Syx\% = 22,736\% para estimativa de biomassa de matéria seca e $\mathrm{R}^{2}=0,962$ e Syx\% = $22,14 \%$ para estimativa de carbono. 
Tabela 3 - Parâmetros e estatísticas dos modelos ajustados para estimativa de biomassa de matéria seca e carbono total de árvores de restauração.

Table 3 - Parameters and statistics of models fitted for estimates of dry biomass and total carbon for restoration trees.

\begin{tabular}{|c|c|c|c|c|c|c|c|c|}
\hline Modelo & $\mathrm{b}_{0}$ & $\mathrm{~b}_{1}$ & $\mathrm{~b}_{2}$ & $\mathrm{~b}_{3}$ & $\mathrm{~b}_{4}$ & FC & $\mathrm{R}_{\text {aj }}^{2}$ & $\mathrm{~S}_{\mathrm{yx}} \%$ \\
\hline \multicolumn{9}{|c|}{ Biomassa para o conjunto total de espécies } \\
\hline 1 & $-2,70205$ & 1,63841 & 1,20481 & - & - & 1,077 & 0,909 & 44,59 \\
\hline 2 & 5,43567 & 0,0359465 & - & - & - & - & 0,917 & 42,49 \\
\hline 3 & $-34,1623$ & 4,92889 & 0,218692 & 0,970921 & 0,0421405 & - & 0,917 & 42,59 \\
\hline 4 & 5,2114 & $-0,069118$ & 0,174175 & 0,037933 & - & - & 0,917 & 42,56 \\
\hline 5 & 17,7008 & $-0,869451$ & 0,0366757 & - & - & - & 0,917 & 42,66 \\
\hline 6 & $-32,6945$ & 5,64542 & $-0,238179$ & 0,042765 & - & - & 0,918 & 42,39 \\
\hline 7 & 19,2349 & $-0,079182$ & 0,0397874 & - & - & - & 0,918 & 42,46 \\
\hline 8 & $-6,6554$ & $-0,12526$ & 0,0478919 & $-0,02611$ & 0,636861 & - & 0,917 & 42,71 \\
\hline \multicolumn{9}{|c|}{ Carbono para o conjunto total de espécies } \\
\hline 1 & $-3,59289$ & 1,65173 & 1,19272 & - & - & 1,083 & 0,905 & 46,51 \\
\hline 2 & 0,107543 & 0,0153481 & - & - & - & - & 0,918 & 43,19 \\
\hline 3 & $-7,38398$ & 2,1792 & - 0,103731 & - 0,613959 & 0,0187293 & - & 0,917 & 43,30 \\
\hline 4 & 5,83112 & - 0,0415504 & 0,0215424 & 0,0171946 & - & - & 0,918 & 43,23 \\
\hline 5 & 11,0288 & - 0,774188 & 0,0159974 & - & - & - & 0,917 & 43,28 \\
\hline 6 & $-8,3121$ & 1,72611 & - 0,091409 & 0,0183343 & - & - & 0,918 & 43,09 \\
\hline 7 & 7,56558 & - 0,042795 & 0,0174239 & - & - & - & 0,918 & 43,03 \\
\hline 8 & $-0,91018$ & - 0,0734437 & 0,022852 & - 0,014833 & 0,284385 & - & 0,917 & 43,32 \\
\hline \multicolumn{9}{|c|}{ Biomassa das espécies de crescimento rápido } \\
\hline 1 & $-2,63354$ & 1,64301 & 1,11309 & - & - & 1,072 & 0,934 & 42,24 \\
\hline 2 & $-26,4403$ & 0,0343808 & - & - & - & - & 0,964 & 31,07 \\
\hline 3 & 86,5176 & 3,98218 & - 0,244609 & $-14,698$ & 0,0462921 & - & 0,966 & 30,18 \\
\hline 4 & 45,6741 & - 0,17908 & - 0,523568 & 0,0462864 & - & - & 0,967 & 29,77 \\
\hline 5 & 61,9501 & - 5,93977 & 0,0386163 & - & - & - & 0,966 & 30,05 \\
\hline 6 & 77,8717 & - 8,03966 & 0,0793322 & 0,036553 & - & - & 0,966 & 30,40 \\
\hline 7 & 4,66253 & - 0,16099 & 0,0416064 & - & - & - & 0,965 & 30,56 \\
\hline 8 & 68,2123 & $-0,07215$ & 0,0291017 & 0,045483 & $-1,4047$ & - & 0,967 & 29,89 \\
\hline \multicolumn{9}{|c|}{ Carbono das espécies de crescimento rápido } \\
\hline 1 & $-3,4906$ & 1,65279 & 1,09078 & - & - & 1,079 & 0,878 & 58,42 \\
\hline 2 & $-14,425$ & 0,014797 & - & - & - & - & 0,961 & 32,97 \\
\hline 3 & 47,4789 & 1,29583 & - 0,099759 & - 7,28272 & 0,0202191 & - & 0,965 & 31,22 \\
\hline 4 & 23,5975 & - 0,088642 & - 0,29164 & 0,0209299 & - & - & 0,967 & 30,64 \\
\hline 5 & 31,0008 & $-3,05258$ & 0,0169737 & - & - & - & 0,965 & 31,36 \\
\hline 6 & 43,1949 & - 4,66086 & 0,0607596 & 0,0153934 & - & - & 0,964 & 31,63 \\
\hline 7 & 0,753086 & - 0,078561 & 0,0183231 & - & - & - & 0,963 & 32,22 \\
\hline 8 & 30,5752 & - 0,055535 & 0,0156097 & 0,0140813 & 0,564433 & - & 0,966 & 30,91 \\
\hline \multicolumn{9}{|c|}{ Biomassa das espécies de crescimento médio } \\
\hline 1 & $-3,2199$ & 1,6085 & 1,46474 & - & - & 1,076 & 0,856 & 45,82 \\
\hline 2 & $-29,548$ & 0,0430519 & - & - & - & - & 0,861 & 44,98 \\
\hline 3 & 58,9394 & 10,8132 & - 0,757142 & $-15,7804$ & 0,0809173 & - & 0,891 & 42,63 \\
\hline 4 & 63,1015 & - 0,51075 & - 0,415946 & 0,0769179 & - & - & 0,892 & 42,05 \\
\hline 5 & 133,883 & - 12,9123 & 0,0581054 & - & - & - & 0,879 & 44,54 \\
\hline 6 & 15,1774 & 0,850032 & - 0,446473 & 0,0684823 & - & - & 0,890 & 42,55 \\
\hline 7 & 23,7081 & - 0,42854 & 0,068412 & - & - & - & 0,893 & 39,53 \\
\hline 8 & 40,048 & - 0,61591 & 0,0962903 & - 0,05095 & 0,461124 & - & 0,891 & 42,43 \\
\hline
\end{tabular}

Revista Árvore, Viçosa-MG, v.35, n.3, Edição Especial, p.679-689, 2011 
Tabela 1 - Cont.

Table 1 - Cont.

\begin{tabular}{|c|c|c|c|c|c|c|c|c|}
\hline \multicolumn{9}{|c|}{ 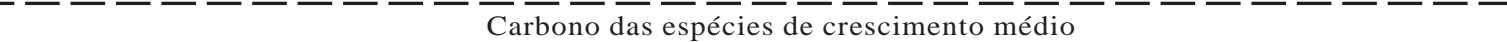 } \\
\hline 1 & $-4,14452$ & 1,61745 & 1,47207 & - & - & 1,083 & 0,851 & 46,83 \\
\hline 2 & $-12,5502$ & 0,0180347 & - & - & - & - & 0,855 & 46,21 \\
\hline 3 & 26,1041 & 4,97797 & - 0,330652 & $-7,28189$ & 0,034413 & - & 0,885 & 41,22 \\
\hline 4 & 27,9515 & - 0,217299 & - 0,192417 & 0,0325782 & - & - & 0,886 & 40,93 \\
\hline 5 & 55,7067 & $-5,3928$ & 0,0243218 & - & - & - & 0,873 & 43,27 \\
\hline 6 & 5,91005 & 0,380447 & - 0,187293 & 0,0286748 & - & - & 0,883 & 41,47 \\
\hline 7 & 9,72811 & - 0,179269 & 0,0286435 & - & - & - & 0,887 & 40,96 \\
\hline 8 & 18,2 & $-0,26178$ & 0,0407726 & $-0,021551$ & 0,178579 & - & 0,884 & 41,30 \\
\hline \multicolumn{9}{|c|}{ Biomassa das espécies de crescimento lento } \\
\hline 1 & $-2,79474$ & 1,85892 & 1,08036 & - & - & 1,049 & 0,982 & 17,81 \\
\hline 2 & 3,78824 & 0,0474763 & - & - & - & - & 0,985 & 16,15 \\
\hline 3 & 17,6174 & $-4,69787$ & 0,195967 & 2,47815 & 0,0409674 & - & 0,983 & 17,20 \\
\hline 4 & 7,15968 & $-0,03743$ & - 0,003905 & 0,0498773 & - & - & 0,983 & 16,93 \\
\hline 5 & 13,6018 & - 0,96208 & 0,0489453 & - & - & - & 0,984 & 16,46 \\
\hline 6 & 21,4902 & $-2,43738$ & 0,0842513 & 0,045897 & - & - & 0,983 & 16,85 \\
\hline 7 & 6,93897 & - 0,03707 & 0,0498089 & - & - & - & 0,984 & 16,50 \\
\hline 8 & $-11,572$ & $-0,16044$ & 0,0888397 & - 0,09468 & 1,00576 & - & 0,985 & 15,95 \\
\hline \multicolumn{9}{|c|}{ Carbono das espécies de crescimento lento } \\
\hline 1 & $-3,71971$ & 1,89002 & 1,06358 & - & - & 1,048 & 0,979 & 18,80 \\
\hline 2 & 1,80768 & 0,0197598 & - & - & - & - & 0,983 & 16,74 \\
\hline 3 & 7,19668 & $-2,0882$ & 0,0963576 & 1,09755 & 0,0162867 & - & 0,981 & 17,87 \\
\hline 4 & 2,63461 & - 0,0075764 & - 0,003759 & 0,0202805 & - & - & 0,983 & 17,12 \\
\hline 5 & 4,52261 & - 0,26616 & 0,0201661 & - & - & - & 0,983 & 17,12 \\
\hline 6 & 8,91194 & - 1,08706 & 0,0468799 & 0,01847 & - & - & 0,982 & 17,51 \\
\hline 7 & 2,42216 & - 0,0072303 & 0,0202147 & - & - & - & 0,983 & 17,15 \\
\hline 8 & $-5,58064$ & - 0,0615244 & 0,0373684 & $-0,041523$ & - & - & 0,984 & 13,06 \\
\hline
\end{tabular}

Tabela 4 -Equações geradas por processo Stepwise para estimativa da biomassa seca e carbono total para árvores de reflorestamentos de restauração com respectivas estatísticas.

Table 4 - Equations obtained by Stepwise process for estimate of dry biomass and total carbon for restoration reforestation trees with respective statistics.

\begin{tabular}{|c|c|c|c|c|}
\hline $\mathrm{N}^{\circ}$ & & FC & $\mathrm{R}^{2}$ & Syx\% \\
\hline \multicolumn{5}{|c|}{ Todas as árvores } \\
\hline 9 & $B S T=-10,9293-0,009982 D q^{2} D m+0,0159518 D q^{2} I d+511,77 G h+0,0049168 I d^{2} D m$ & - & 0,937 & 30,06 \\
\hline 10 & $\ln B S T=-2,07402-0,0000094 D m^{2} I d+0,000942267 D q I d+1,27013 \ln D q h$ & 1,060 & 0,954 & 31,72 \\
\hline 9 & $C T=-9,06007+214,071 G h-0,003428 D q^{2} D m+0,00440131 D q^{2} I d$ & - & 0,957 & 31,19 \\
\hline 10 & $\ln C T=4,73036+0,000628125 D q I d+0,780894 \ln G h$ & 1,065 & 0,910 & 45,31 \\
\hline \multicolumn{5}{|c|}{ Árvores de crescimento rápido } \\
\hline 9 & $B S T=-3,99732+353,976 G h+0,0168609 I d^{2} h$ & - & 0,971 & 28,00 \\
\hline 10 & $\ln B S T=1,03747+0,00286391 G+1,83393 \ln D q^{2} h$ & 1,068 & 0,963 & 31,50 \\
\hline 9 & $C T=-3,02986+145,866 G h+0,00856095 I d^{2} h$ & - & 0,971 & 28,54 \\
\hline 10 & $\ln C T=-1,51691+0,0014982 D q h+1,68616 \ln D q$ & 1,070 & 0,890 & 55,65 \\
\hline \multicolumn{5}{|c|}{ Árvores de crescimento médio } \\
\hline 9 & $B S T=30,1977-494,53 G^{2} D m+1346,73 G^{2} I d+24,563 h^{2} G$ & - & 0,973 & 19,64 \\
\hline 10 & $\ln B S T=0,3556+0,00385906 h^{2}+0,000651916 D q I d+1,51608 \ln D q$ & 1,062 & 0,948 & 27,46 \\
\hline 9 & $C T=12,5279-214,646 G^{2} D m+584,089 G^{2} I d+10,1808 h^{2} G$ & - & 0,973 & 20,04 \\
\hline 10 & $\ln C T=1,23797+0,003775 h^{2}+0,000695783 D q I d+1,51343 \ln D q$ & 1,068 & 0,944 & 28,63 \\
\hline \multicolumn{5}{|c|}{ Árvores de crescimento lento } \\
\hline 9 & $B S T=26,2097-0,22735 D m^{2}+0,0711221 D q^{2} h-0,01933 h^{2} I d$ & - & 0,991 & 12,46 \\
\hline 10 & $\ln B S T=-2,46958+1,26379 \ln D q^{2}-0,318736 \ln I d^{2}+0,490108 \ln h^{2}$ & 1,031 & 0,968 & 23,19 \\
\hline 9 & $C T=5,54962-0,0826953 D^{2}+316,458 G h$ & - & 0,988 & 14,35 \\
\hline 10 & $\ln C T=-3,71971+1,89002 \ln D q+1,06358 \ln h$ & 1,048 & 0,979 & 18,80 \\
\hline
\end{tabular}

BST = Biomassa de matéria seca total; e CT = Carbono total. 

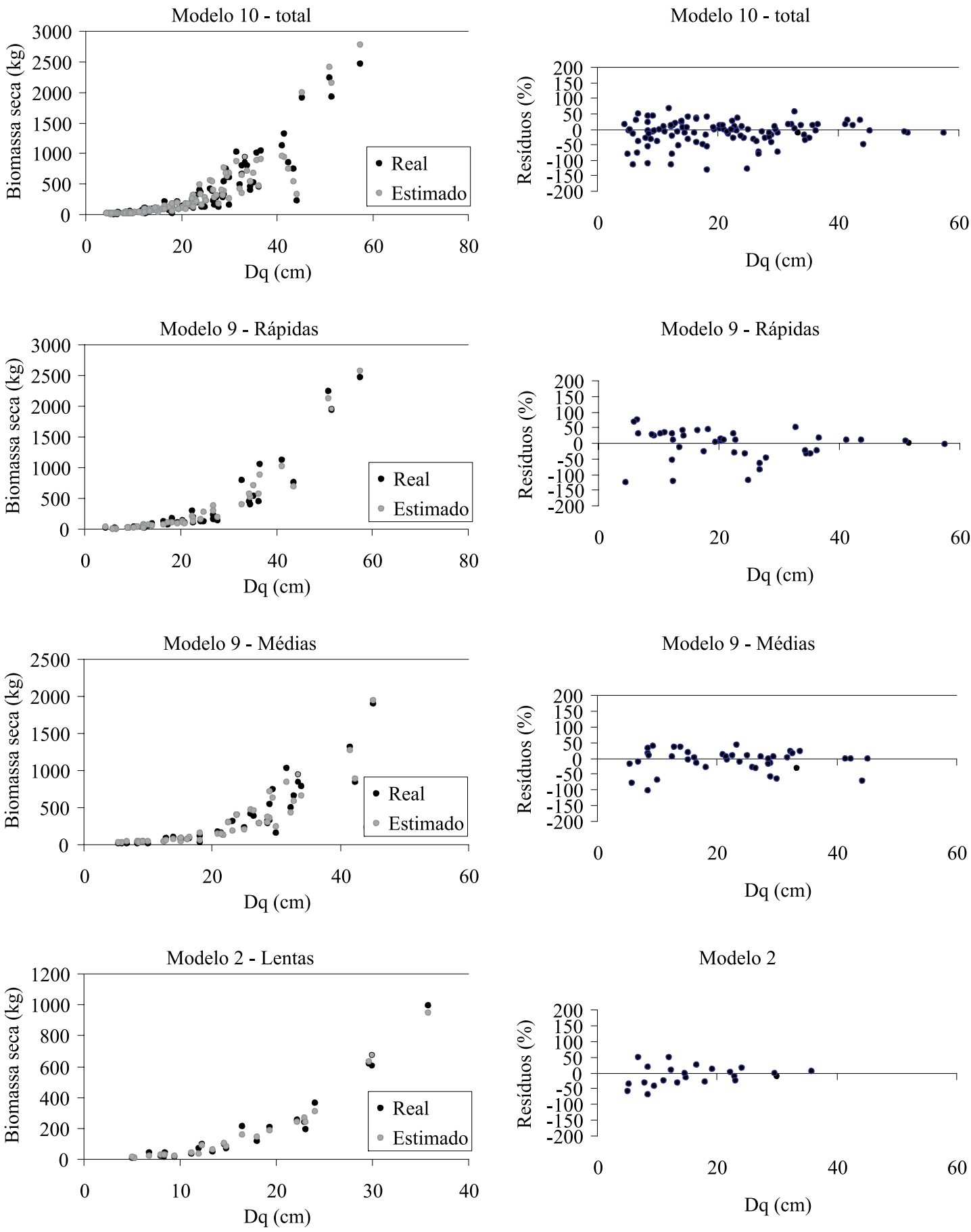

Modelo 2

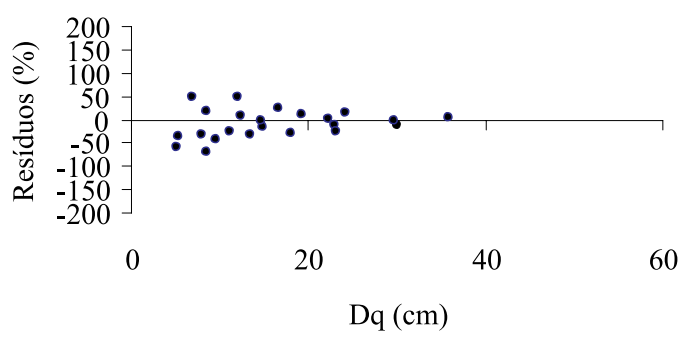

Figura 1 - Distribuições gráficas dos valores reais de biomassa de matéria seca e de resíduos, em função do diâmetro quadrático, de alguns modelos ajustados para o conjunto total das espécies e por ritmo de crescimento de árvores de reflorestamento de restauração.

Figure 1 - Graph distribution of true values of dry biomass and residues in function of quadratic diameter, for some adjusted models for total set of species and per growth rate in restoration reforestation trees.

Revista Árvore, Viçosa-MG, v.35, n.3, Edição Especial, p.679-689, 2011 

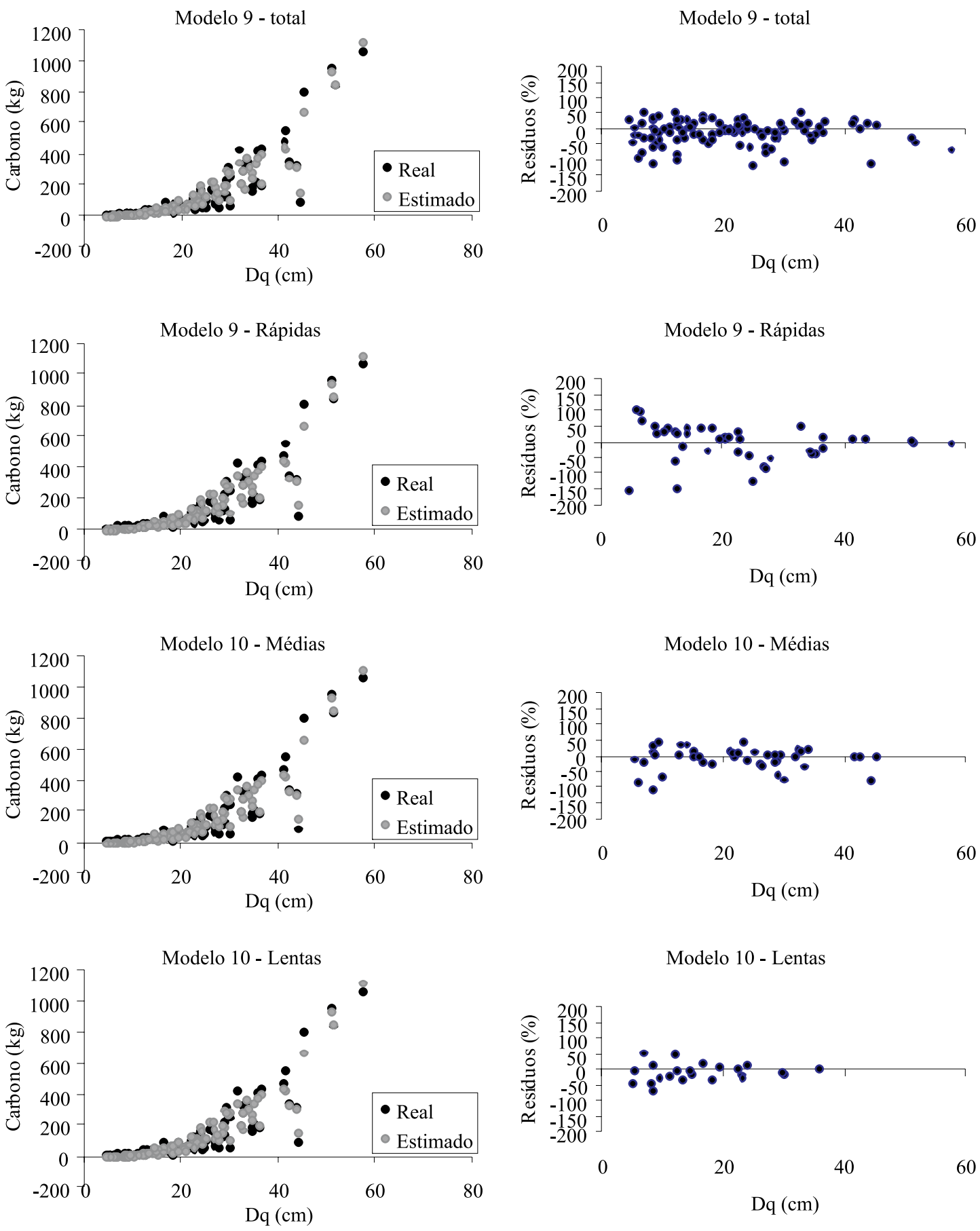

Modelo 10 - Lentas

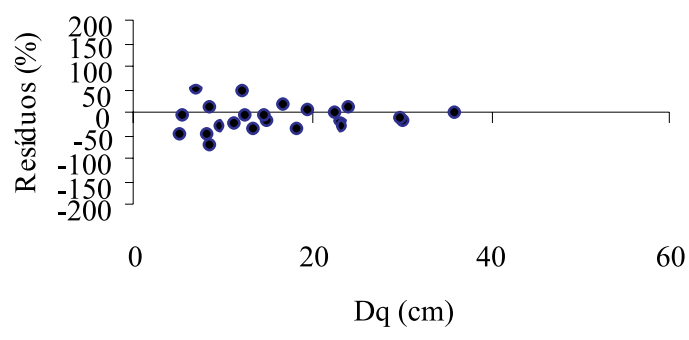

Figura 2 - Distribuições gráficas dos valores reais de carbono total e de resíduos, em função do diâmetro quadrático, de alguns modelos ajustados para o conjunto total das espécies e por ritmo de crescimento de árvores de reflorestamento de restauração.

Figure 2 - Graph distribution of true values of total carbon and residues in function of the quadratic diameter, for some models adjusted for total species set and per growth rate in restoration reforestation trees. 


\section{CONCLUSÕES}

Os modelos testados e ajustados apresentaram boa qualidade, permitindo estimativas apropriadas para as variáveis biomassa de matéria seca e carbono total em árvores de reflorestamentos de restauração florestal, nas mesmas condições da região de amostragem.

A estratificação por ritmo de crescimento das árvores permitiu melhorar o desempenho dos modelos alométricos.

\section{AGRADECIMENTOS}

Ao Projeto de Recuperação de Matas Ciliares da Secretaria de Meio Ambiente do Estado de São Paulo, pelo financiamento deste estudo.

\section{REFERÊNCIAS}

BROWN, S.; GILLESPIE, A. J. R.; LUGO, A. E. Biomasss estimation methods for tropical forests with applications to forest inventory data.

Forest Ecology, v.35, p.881-902, 1989.

CALDEIRA, M. V. W.; SOARES, R. V.;

WATZLAWICK, L. F. Estimativa de Biomassa em Espécies Arbóreas da Floresta Ombrófila Mista Montana - General Carneiro-PR. Biomassa \& Energia, v.1, p.291-310, 2004.

CHAMBERS, J. Q. et al. Tree damage, allometric relationships, and above ground net primary production in central Amazon forest. Forest Ecology and Management, v. 152, n.1, p. 73-84, 2001.

DELITTI, W. B. C.; PAUSAS, J. G.; MOREIRABURGER, D. M. Belowground biomass seasonal variation in two Neotropical savannahs (Brazilian Cerrados) with different fire histories. Annals of Forest Science, v.58, p.713-721, 2001.

DRAPER, N. R.; SMITH, H. Applied regression analysis. 2.ed. New York: John Wiley \& Sons, 1980.709p.

DURIGAN, G. et al. A flora arbusto-arbórea do médio Paranapanema: base para restauração dos ecossistemas naturais. In: DURIGAN, G.; BÔAS, V. O. Pesquisas em conservação e recuperação ambiental no Oeste Paulista. São Paulo: Páginas e Letras, 2004. p.199-240.
ENGEL, V. L., PARROTA, J. A. Definindo a restauração ecológica: tendências e perspectivas mundiais. In: KAGEYAMA, P. Y. et al. (Orgs.) Restauração ecológica de ecossistemas naturais. Botucatu: FEPAF, 2003. p.1-26.

HIGUCHI, N.; CARVALHO JR, J. A. Fitomassa e conteúdo de carbono de espécies arbóreas da Amazônia. In: Emissão $\times$ Seqüestro de CO2 - Uma nova oportunidade de negócios para o Brasil, 1994. Rio de Janeiro: 1994. p.125-153.

HIGUCHI, N. et al. Biomassa da parte aérea da vegetação da floresta tropical úmida de terra-firme da Amazônia brasileira. Manaus. Acta Amazônica, v.28, n.1, p.153-166, 1998.

HIGUCHI, N. et al. Dinâmica e balanço do carbono da vegetação primária da Amazônia Central. Floresta, v.34 p.295-304, 2004.

KOEHLER, H. S. et al. Fontes de erros nas estimativas de biomassa e carbono fixado na floresta ombrófila mista. Biomassa \& Energia, v.2, n.1, p.69-77, 2005.

MACDICKEN, K. G.; WOLF, G. V.; BRISCOE, C. B. Standard research methods for multipurpose tree and shrubs. Arlington: Winrock International Institute for Agricultural Development/ICRAF, 1991. 92p.

MELO, A. C. G.; DURIGAN, G. Fixação de carbono em reflorestamentos de matas ciliares no Vale do Paranapanema, SP, Brasil. Scientia Forestalis, v.71, p.149-154, 2006.

MOREIRA-BURGER, D.; DELITTI, W. B. C. Fitomassa epigéa da mata ciliar do rio MogiGuaçu, Itapira - SP. Revista Brasileira de Botânica, v.22, p.429-435, 1999.

NELSON, B. W. et al. Allometric regresions for improved estimate of seondary forest biomass in the central Amazon. . Forest Ecology and Management, v.117, p.149-167, 1999.

REZENDE, A. V. et al. Comparação de modelos matemáticos para estimativa do volume, biomassa e estoque de carbono da vegetação lenhosa de um cerrado sensu stricto em Brasília, DF.

Scientia Forestalis, n.71, p.65-76, 2006. 
SALLIS, S. M. et al. Estimating the aboveground biomass and wood volume of savanna woodlands in Brazil Pantanal wetlands base don allometric correlations. Forest Ecology and Management, v.228, p.61-68, 2006.

SANQUETTA C. R. et al. Relações individuais de biomassa e conteúdo carbono em plantações de Araucaria angustifolia e Pinus taeda no sul do estado do Paraná, Brasil. In: SIMPÓSIO LATINO AMERICANO SOBRE MANEJO FLORESTAL, 2., 2001, Santa Maria. Brasil. Anais... Santa Maria: UFSM/CCR/PPGEF, 2001. p.415-427.

SANQUETTA, C. R. Métodos de determinação de biomassa florestal. In: SANQUETTA, C. R. et al. As florestas e o carbono. Curitiba: Universidade Federal de Paraná, 2002. p.119-140.

SANQUETTA, C. R. et al. Proposta metodológica para quantificação e monitoramento do carbono estocado em florestas plantadas. In:

SANQUETTA, C. R. \& ZILIOTTO, M. A. B. (Eds.) Carbono: ciência e mercado global. Curitiba: UFPR / ECOPLAN, 2004. p.240-265.

SANTOS, J. et al. Modelos estatísticos para estimar a fitomassa acima do nível do solo da floresta tropical úmida da Amazônia Central. Revista Árvore, v.25, n.4, p.445-454, 2001.
SANTOS, K. et al. Equações volumétricas por classe diamétrica para algumas espécies folhosas da Floresta Ombrófila Mista no Paraná, Brasil. Revista de Ciências Exatas e Naturais, v.8, v.1, p.99-112, 2006.

SCOLFORO, J. R. S. et al. Estimativas de volume, peso seco, peso de óleo e quantidade de mourões para candeia (Eremanthus erythropappus (DC.) MacLeish). Cerne, v.10, n.1, p.87-102. jan./jun. 2004.

SILVA, G. C.; SAMPAIO, E. V. S. B. Biomassas de partes aéreas em plantas de caatinga. Revista Árvore, v.32, n.3, p.567-575, 2008.

SILVEIRA, E. R.; DURIGAN, G. Recuperação das matas ciliares: estrutura da floresta e regeneração natural aos 10 anos em diferentes modelos de plantio na Fazenda Canaçu, Tarumã, SP. In: VILAS BOAS, O.; DURIGAN, G. Pesquisas em conservação e recuperação ambiental no oeste paulista: resultados da cooperação Brasil/Japão. São Paulo: Páginas e Letras, 2004. p.347-370.

URBANO, E. Estimativa da biomassa aérea e do peso de carbono fixado em árvores de bracatingais nativos da região metropolitana de Curitiba. 2006. 84f. Dissertação (Mestrado em Engenharia Florestal) - Universidade Federal do Paraná, Curitiba, 2006. 
